\title{
DNA Methylation Status of SHOX-Flanking CpG Islands in Healthy Individuals and Short Stature Patients with Pseudoautosomal Copy Number Variations
}

\author{
Kenichiro Ogushia,b Atsushi Hattoria, crina Suzuki ${ }^{a}$ Hirohito Shima ${ }^{a}$ \\ Masako Izawa $^{d}$ Hideaki Yagasaki $^{e}$ Reiko Horikawa ${ }^{f}$ Kimiaki Uetake $^{g}$

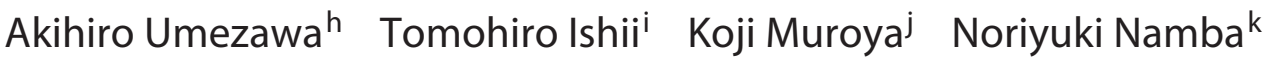 \\ Toshiaki Tanakal Yasuhiro Hirano $^{m}$ Hitoshi Yamamoto $^{b}$ Shun Soneda ${ }^{b}$ \\ Keiko Matsubara ${ }^{a}$ Masayo Kagami ${ }^{a}$ Mami Miyado ${ }^{a}$ Maki Fukami ${ }^{a}$

\begin{abstract}
aDepartment of Molecular Endocrinology, National Research Institute for Child Health and Development, Tokyo,

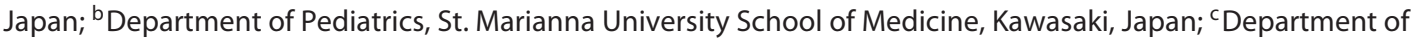
Advanced Pediatric Medicine, Tohoku University School of Medicine, Tokyo, Japan; ${ }^{\mathrm{d} D e p a r t m e n t}$ of Pediatric

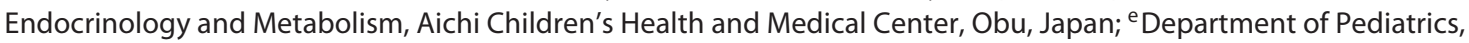
Faculty of Medicine, University of Yamanashi, Chuo, Japan; ' Division of Endocrinology and Metabolism, National Center for Child Health and Development, Tokyo, Japan; ${ }^{9}$ Department of Pediatrics, Obihiro Kosei General Hospital, Obihiro, Japan; ' Department of Reproductive Biology, National Research Institute for Child Health and Development, Tokyo, Japan; 'Department of Pediatrics, Yokohama Municipal Citizen's Hospital, Yokohama, Japan; 'Department of Endocrinology and Metabolism, Kanagawa Children's Medical Center, Yokohama, Japan; kDepartment of Pediatrics, Osaka Hospital, Japan Community Healthcare Organization (JCHO), Osaka, Japan;

'Tanaka Growth Clinic, Tokyo, Japan; 'm Department of Pediatrics, Hiratsuka City Hospital, Hiratsuka, Japan
\end{abstract}

\section{Keywords}

Copy number variation · CpG island · DNA methylation . Idiopathic short stature - Leri-Weill dyschondrosteosis

\begin{abstract}
SHOX resides in the short arm pseudoautosomal region (PAR1) of the sex chromosomes and escapes $X$ inactivation. SHOX haploinsufficiency underlies idiopathic short stature (ISS) and Leri-Weill dyschondrosteosis (LWD). A substantial percentage of cases with SHOX haploinsufficiency arise from pseudoautosomal copy number variations (CNVs) involving putative enhancer regions of SHOX. Our previous study using peripheral blood samples showed that some CpG dinu-
\end{abstract}

cleotides adjacent to SHOX exon 1 were hypomethylated in a healthy woman and methylated in a woman with gross $X$ chromosomal rearrangements. However, it remains unknown whether submicroscopic pseudoautosomal CNVs cause aberrant DNA methylation of SHOX-flanking CpG islands. In this study, we examined the DNA methylation status of SHOX-flanking CpG islands in 50 healthy individuals and 10 ISS/LWD patients with pseudoautosomal CNVs. In silico analysis detected 3 CpG islands within the $20-\mathrm{kb}$ region from the translation start site of SHOX. Pyrosequencing and bisulfite sequencing of genomic DNA samples revealed that these CpG islands were barely methylated in peripheral blood cells and cultured chondrocytes of healthy individuals, as well as in peripheral blood cells of ISS/LWD patients

\section{KARGER}

(c) 2019 S. Karger AG, Basel

E-Mail karger@karger.com

www.karger.com/cgr 
with pseudoautosomal CNVs. These results, in conjunction with our previous findings, indicate that the DNA methylation status of SHOX-flanking CpG islands can be affected by gross $\mathrm{X}$-chromosomal abnormalities, but not by submicroscopic CNVs in PAR1. Such CNVs likely disturb SHOX expression through DNA methylation-independent mechanisms, which need to be determined in future studies.

(c) 2019 S. Karger AG, Basel

SHOX (NM_000451.3) is a growth-controlling gene located in the short arm pseudoautosomal region (PAR1) of the X and Y chromosomes [Rao et al., 1997]. SHOX escapes X chromosome inactivation (XCI) and exhibits biallelic expression in both males and females [Carrel and Willard, 2005]. SHOX haploinsufficiency accounts for 1.9-22.2\% of idiopathic short stature (ISS; OMIM 300582) and $33.9-100 \%$ of Leri-Weill dyschondrosteosis (LWD; OMIM 127300) [Schneider et al., 2005; Funari et al., 2010; Benito-Sanz et al., 2012; Rosilio et al., 2012; Poggi et al., 2015]. The most common genetic cause of SHOX haploinsufficiency is copy number variations (CNVs) involving SHOX and/or its putative enhancer regions [BenitoSanz et al., 2006; Chen et al., 2009; Rosilio et al., 2012]. To date, 7 highly conserved noncoding DNA elements (CNEs) with cis-regulatory activity have been reported as putative enhancer regions of SHOX [Fukami et al., 2006; Chen et al., 2009; Durand et al., 2010; Verdin et al., 2015]. Furthermore, since various pseudoautosomal CNVs outside of known CNEs were identified in ISS/LWD patients [Bunyan et al., 2014; Sandoval et al., 2014; Fukami et al., 2015], additional SHOX enhancers may reside in PAR1. Submicroscopic CNVs involving known CNEs lead to a phenotype similar to that of intragenic mutations [Flanagan et al., 2002; Benito-Sanz et al., 2012; Verdin et al., 2015], indicating that copy number changes of the enhancers can completely abolish SHOX expression. Indeed, Flanagan et al. [2002] documented monoallelic expression of SHOX in skeletal tissues of a patient with a SHOX downstream deletion.

CpG islands often reflect transcriptional levels of adjacent genes [Deaton and Bird, 2011]. Typically, genes escaping XCI are coupled with hypomethylated CpG islands in genomic DNA samples of both sexes, while genes subjected to XCI frequently exhibit substantial methylation of nearby $\mathrm{CpG}$ islands exclusively in female samples [Plath et al., 2002]. Since SHOX is an XCI escapee gene [Carrel and Willard, 2005], it may also be accompanied by hypomethylated $\mathrm{CpG}$ islands. Indeed, we have previously shown that $12 \mathrm{CpG}$ dinucleotides adjacent to SHOX exon 1 were hypomethylated in peripheral blood cells of a healthy woman [Suzuki et al., 2016]. Furthermore, in that study, we found that the same CpG dinucleotides were markedly methylated in peripheral blood cells of a woman with short stature and catastrophic X chromosomal rearrangements with a $46, \mathrm{X}, \operatorname{der}(\mathrm{X})(\mathrm{pter} \rightarrow \mathrm{p} 22.31::$ $\mathrm{q} 22.1 \rightarrow \mathrm{q} 10:: \mathrm{q} 10 \rightarrow \mathrm{q} 22.1:: \mathrm{p} 22.31 \rightarrow \mathrm{pter})$ karyotype. These findings provided the first indication that $\mathrm{X}$-chromosomal rearrangements can affect DNA methylation of SHOXflanking CpG islands, which are otherwise hypomethylated. However, because only a small number of CpG dinucleotides in only 2 individuals were examined in that study, the results need to be validated. Moreover, our data raised an important question as to whether pseudoautosomal CNVs lead to ISS/LWD by causing abnormal DNA methylation of the CpG islands. In the present study, we analyzed the DNA methylation status in several healthy individuals and patients with ISS/LWD and submicroscopic CNVs in PAR1.

\section{Materials and Methods}

Identification of SHOX-Flanking CPG Islands

We performed in silico analysis to identify $\mathrm{CpG}$ islands in the SHOX-flanking region. A $20-\mathrm{kb}$ genomic interval around the translation start site of SHOX (chrX:611,501-631,500) was analyzed using the default settings of the UCSC Genome Browser (www.genome.ucsc.edu/; hg38, build 38).

\section{Clinical Samples}

Peripheral blood samples were obtained from 9 healthy adults with normal height ( 4 males and 5 females) and 40 children without apparent short stature ( 20 boys and 20 girls). We also examined a genomic DNA sample obtained from cultured chondrocytes established from a surgical specimen of a boy with polydactyly [Nasu et al., 2015].

Furthermore, we obtained DNA samples from 10 patients with ISS or LWD and submicroscopic CNVs in PAR1 (Table 1). CNVs in these patients were detected by multiplex ligation-dependent amplification (SALSA P018-G1, MRC-Holland, Netherlands) or comparative genomic hybridization using a custom-made array (8 $\times 60$ k format, Agilent Technologies, Santa Clara, CA, USA). CNVs in patients 1-5 encompassed 1 or more of the known CNEs, while those in patients 6-10 involved genomic regions outside of the CNEs (Fig. 1). Seven of the 10 patients were identified through our previous analysis of 328 patients with ISS or LWD [Shima et al., 2016].

\section{DNA Methylation Analysis of the SHOX-Flanking CpG}

Islands

We examined the DNA methylation status of 3 SHOX-flanking CpG islands (CpG islands 1, 2, and 3). Genomic DNA samples were treated with bisulfite using the EZ DNA Methylation-Gold kit (Zymo Research, Irvine, CA, USA). From each CpG island, a DNA fragment containing 6 or more CpG dinucleotides was PCR- 


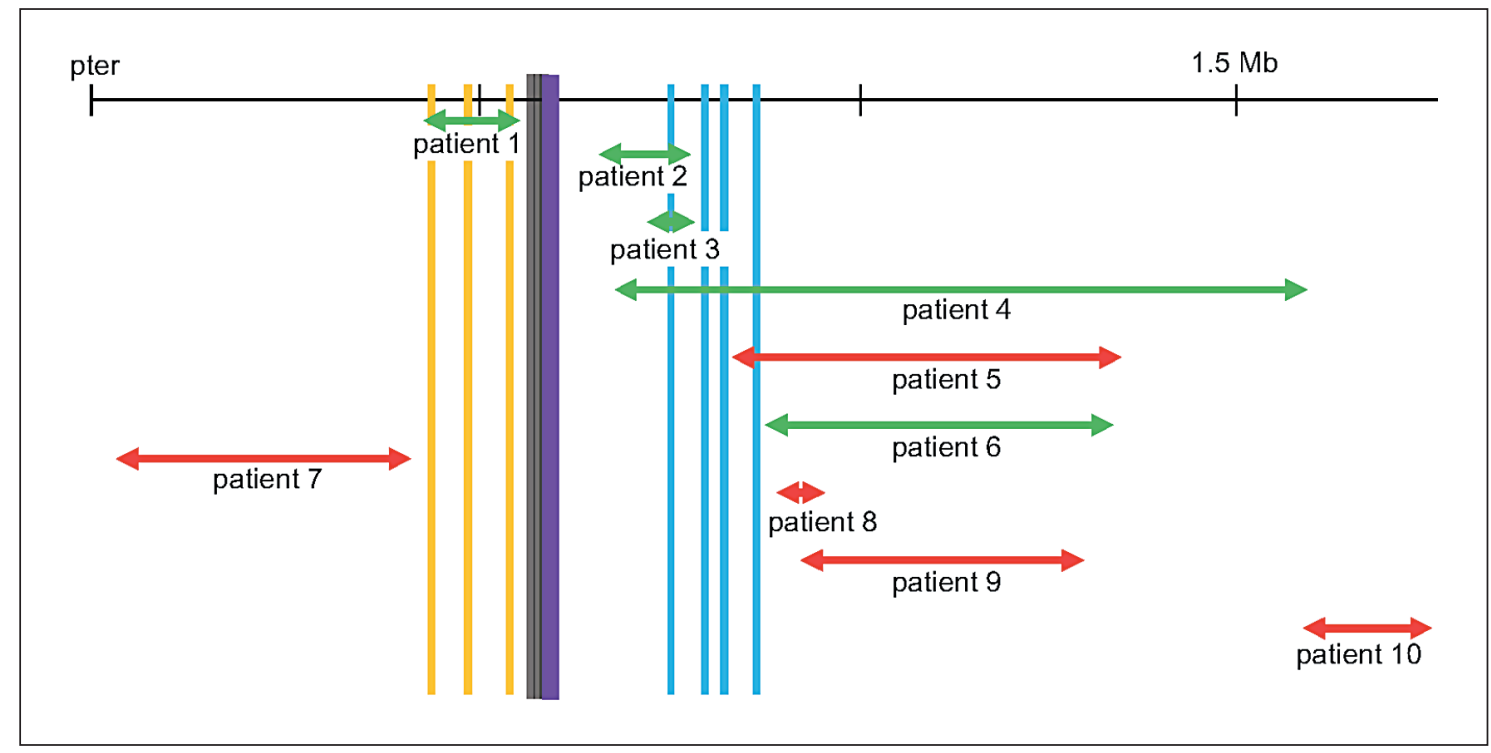

Fig. 1. Genomic position of copy number variations in 10 patients. The purple and gray bars indicate SHOX exons and CpG islands, respectively. The yellow and blue bars denote the putative upstream and downstream enhancer regions, respectively. The green arrows indicate deleted regions, while the red arrows denote amplified regions. pter, short arm telomere.

Table 1. Clinical and molecular findings of patients with pseudoautosomal CNVs

\begin{tabular}{|c|c|c|c|c|c|c|c|c|c|}
\hline Patient & Sex & $\begin{array}{l}\text { Age, } \\
\text { years }\end{array}$ & $\begin{array}{l}\text { Height, } \\
\text { SDS }\end{array}$ & $\begin{array}{l}\text { Skeletal } \\
\text { deformity }\end{array}$ & CNV type & $\begin{array}{l}\text { Size of } \\
\mathrm{CNV}^{\mathrm{a}}, \mathrm{kb}\end{array}$ & $\begin{array}{l}\text { Genomic position of } \\
\mathrm{CNV}^{\mathrm{a}}\end{array}$ & Affected SHOX enhancer ${ }^{\mathrm{b}}$ & $\begin{array}{l}\text { Previous } \\
\text { report }^{c}\end{array}$ \\
\hline 1 & M & 4.0 & -5.0 & None & Deletion & 128 & $428,003-556,839$ & CNE-5, CNE-3, CNE-2 & Case 4 \\
\hline 3 & $\mathrm{~F}$ & 11.0 & -2.4 & None & Deletion & 35 & $751,623-787,295$ & CNE4 & Case 8 \\
\hline 4 & M & 16.0 & -3.0 & Madelung & Deletion & 920 & $682,821-1,603,243$ & CNE4, CNE5, ECR1, ECS4/CNE9 & Case 7 \\
\hline 5 & $\mathrm{~F}$ & 4.7 & -2.5 & None & Duplication & 517 & $838,550-1,355,761$ & ECS4/CNE9 & Case 15 \\
\hline 8 & $\mathrm{~F}$ & 7.2 & -3.3 & None & Duplication & 23 & $894,185-918,040$ & None & \\
\hline 9 & M & 6.5 & -2.0 & None & Duplication & 376 & $928,644-1,305,631$ & None & Case 16 \\
\hline 10 & $\mathrm{~F}$ & 13.0 & -4.0 & None & Duplication & 171 & $1,592,201-1,763,328$ & None & \\
\hline
\end{tabular}

CNE, highly conserved noncoding DNA elements; CNV, copy number variation; ECR, evolutionarily conserved region; ECS, evolutionarily conserved sequence; F, female; $\mathrm{M}$, male; SDS, standard deviation score.

a Minimal size of each CNV estimated from the results of array-based comparative genomic hybridization (hg38, build 38).

b Genomic position: CNE-5, chrX:437,622 - 438,171; CNE-3, chrX:499,544-499,929; CNE-2, chrX:555,875-556,494; CNE4, chrX:753,350-754,005; CNE5, chrX:790,090-791,115; ECR1, chrX:819,845-820,500; and ECS4/CNE9, chrX:874,011-874,813.

${ }^{c}$ Previous report: Shima et al. [2016].

amplified (primer sequences are available upon request). The PCR products were subjected to pyrosequencing on PyroMark Q24 (QIAGEN, Valencia, CA, USA). We calculated the methylation index of each CpG dinucleotide by the PyroMark Q24 analysis software.

To confirm the results of pyrosequencing, we analyzed the methylation status of additional CpG dinucleotides in CpG island
1 by bisulfite sequencing. PCR products containing $12 \mathrm{CpG}$ dinucleotides were subcloned using the TOPO TA Cloning Kit (Life Technologies, Carlsbad, CA, USA) and subjected to direct sequencing. The methods were reported previously [Suzuki et al., 2016]. The results of the 10 patients were compared with those of 2 healthy children. We also analyzed a sample obtained from chondrocytes of a healthy boy. 


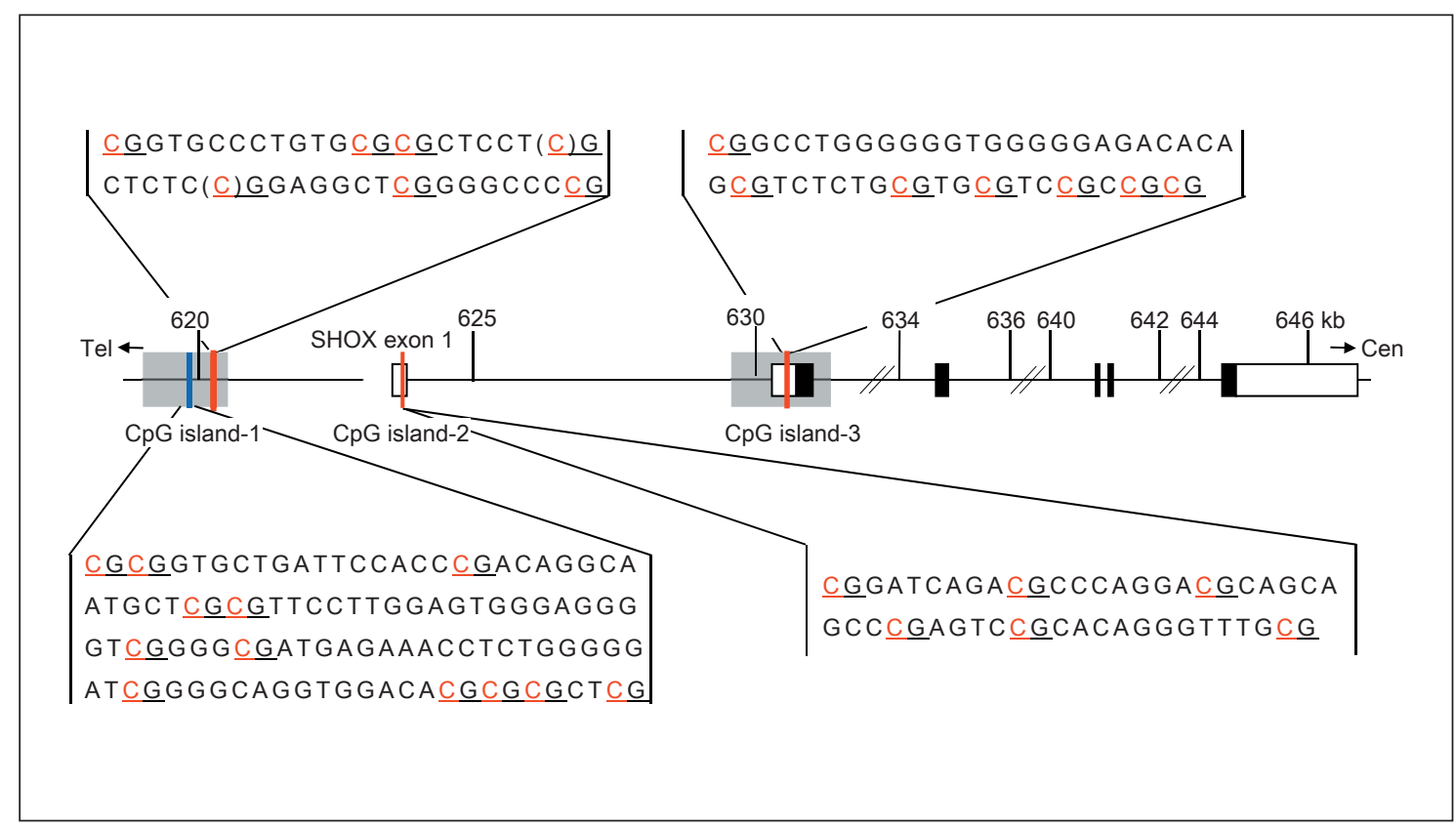

Fig. 2. Genomic structure of $S H O X$ and its flanking CpG islands. The white and black boxes indicate untranslated and translated SHOX exons, respectively. Three CpG islands (CpG islands 1, 2, and 3; shown as gray boxes) are identified within a $20-\mathrm{kb}$ region around the transcription start site of SHOX. CpG dinucleotides in each island are underlined. Red and blue lines depict the target re-

\section{Results}

\section{Identification of SHOX-Flanking CpG Islands}

Three CpG islands were identified in the SHOX-flanking region (CpG island 1 at chrX:619,008-620,527; CpG island 2 at chrX:623,829-624,591; and CpG island 3 at chrX:629,695-631,462) (Fig. 2). These islands were 7631,768 bp in size and encompassed $66-163 \mathrm{CpG}$ dinucleotides.

\section{DNA Methylation Analysis of the SHOX-Flanking CpG Islands}

We analyzed the DNA methylation indexes of 6 or 7 CpG dinucleotides from each CpG island (Fig. 3). CpG islands 1, 2, and 3 were hypomethylated in blood samples of control adults and children. Likewise, these islands were barely methylated in cultured chondrocytes. Methylation indexes of the chondrocytes were almost comparable to or slightly lower than those of blood samples in most $\mathrm{CpG}$ dinucleotides examined.

Furthermore, CpG islands 1, 2, and 3 were hypomethylated in patients $1-10$ with pseudoautosomal CNVs. Actually, methylation indexes of all tested $\mathrm{CpG}$ dinucleo- gions of pyrosequencing and bisulfite sequencing analyses, respectively. Analyzed cytosines are shown in red. Of these, 2 cytosines in $\mathrm{CpG}$ island 1 (shown in parentheses) are not included in the results, because of the poor reproducibility of the methylation analysis. The numbers indicate the genomic distance from pter (hg38, build 38). Tel, short arm telomere; Cen, centromere.

tides in patients 1-10 completely overlapped with those of control individuals.

The results of pyrosequencing were confirmed by bisulfite sequencing in $\mathrm{CpG}$ island 1 (Fig. 4). The $12 \mathrm{CpG}$ dinucleotides were invariably hypomethylated in blood cell-derived genomic DNA samples obtained from healthy individuals and patients $1-10$, as well as in the chondrocyte-derived sample obtained from a healthy boy.

\section{Discussion}

We identified $3 \mathrm{CpG}$ islands around SHOX. Pyrosequencing and bisulfite sequencing of DNA samples revealed that these $\mathrm{CpG}$ islands were barely methylated in peripheral blood cells and cultured chondrocytes of healthy individuals. These results are consistent with the current understanding that SHOX escapes XCI [Carrel and Willard, 2005; Berletch et al., 2011]. Notably, DNA methylation indexes of blood cells and chondrocytes mostly overlapped, except for some $\mathrm{CpG}$ dinucleotides for which chondrocytes showed somewhat lower indexes 


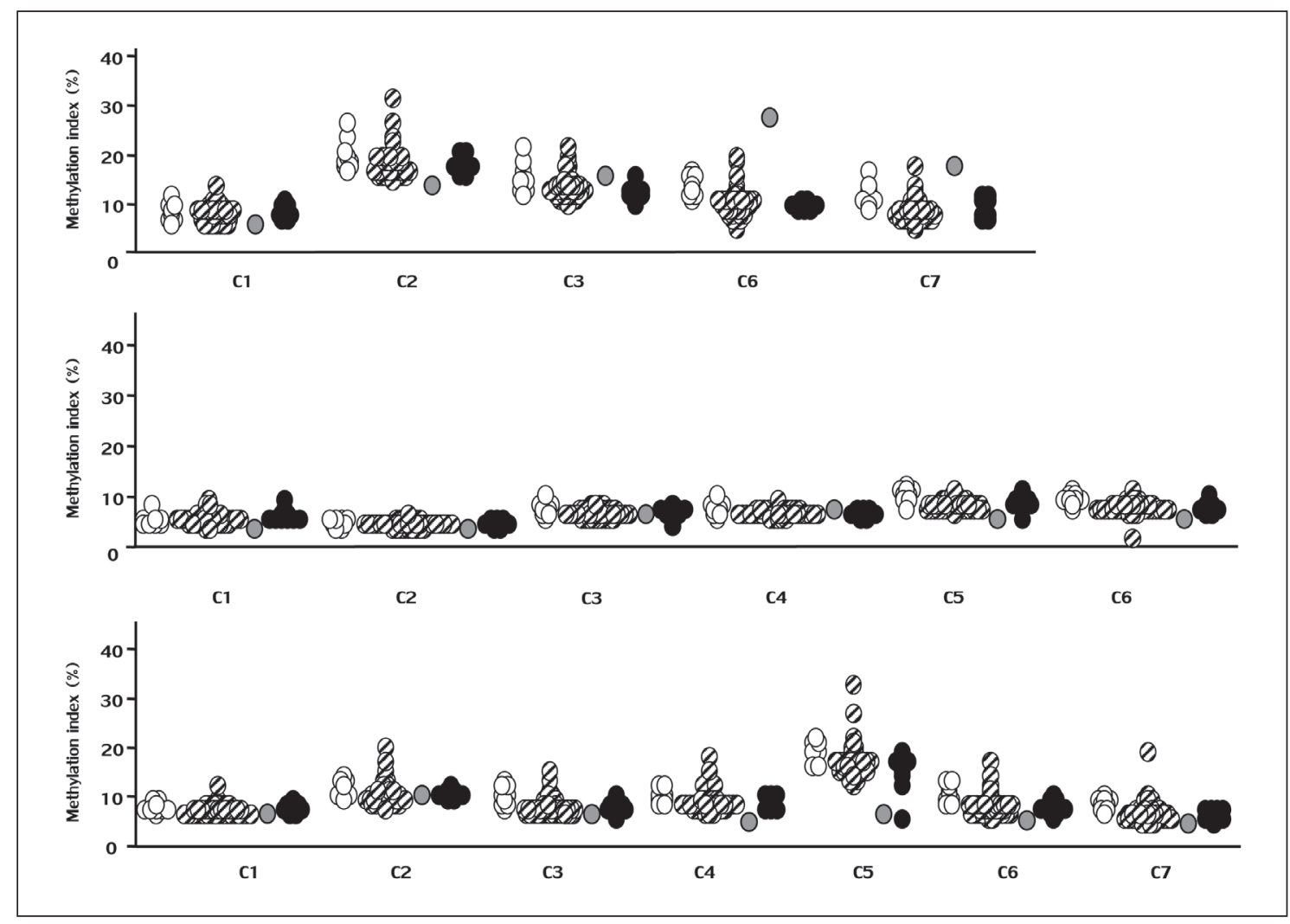

Fig. 3. Results of pyrosequencing. $\mathrm{C} 1-\mathrm{C} 7$ indicate the analyzed cytosines in CpG island 1 (upper panel), CpG island 2 (middle panel), and $\mathrm{CpG}$ island 3 (lower panel). Two cytosines in CpG island 1 ( $\mathrm{C} 4$ and $\mathrm{C} 5)$ are not included in the results because of the poor reproducibility of the methylation analysis. White, striped, and gray circles indicate healthy adults, healthy children, and chondrocytes, respectively. Black circles depict short stature patients with pseudoautosomal copy number variations. than blood cells did (Fig. 3, 4). It is known that SHOX is strongly expressed in osteogenic tissues and weakly expressed in most other tissues [Clement-Jones et al., 2000]. Similar methylation patterns in peripheral blood cells and chondrocytes suggest that tissue-specific expression of SHOX is not dependent on the DNA methylation status of adjacent $\mathrm{CpG}$ islands.

Furthermore, methylation indexes of SHOX-flanking CpG islands were comparable between unaffected individuals and patients with ISS/LWD and pseudoautosomal CNVs. It is worth mentioning that our subjects included patients $1-5$ with CNE-containing CNVs. In particular, patient 4 carried a deletion downstream of $S H O X$, which is predicted to abolish SHOX expression [Flanagan et al., 2002]. The results of the present study indicate that copy number changes of SHOX enhancers do not alter the DNA methylation status of $S H O X$-flanking CpG islands. Thus, pseudoautosomal CNVs may cause SHOX haploinsufficiency by some other mechanisms, such as defective binding of transcription factors to SHOX enhancers or perturbed physical interaction between the SHOX promoter and enhancers.

In our previous study, we detected marked hypermethylation of several $\mathrm{CpG}$ dinucleotides in $\mathrm{CpG}$ island 1 in peripheral blood cells of a woman with gross X-chromosomal rearrangements [Suzuki et al., 2016]. The woman carried a $48-\mathrm{Mb}$ duplication involving SHOX and the $\mathrm{X}$ inactivation-specific transcript (XIST) and other genes, and a $107-\mathrm{Mb}$ deletion involving several genes. Increased DNA methylation in this woman may reflect abnormal XCI for the entire X chromosome, because she had an additional copy of XIST [Deaton and Bird, 2011]. Alternatively, the decreased physical distance between SHOX-flanking CpG islands and XIST may have induced aberrant methylation of these islands. Further analyses of women with various types of X-chromosomal rearrangements will clarify the factors that affect the DNA methylation status of SHOX-flanking CpG islands.
60

Cytogenet Genome Res 2019;158:56-62 DOI: $10.1159 / 000500468$
Ogushi et al. 


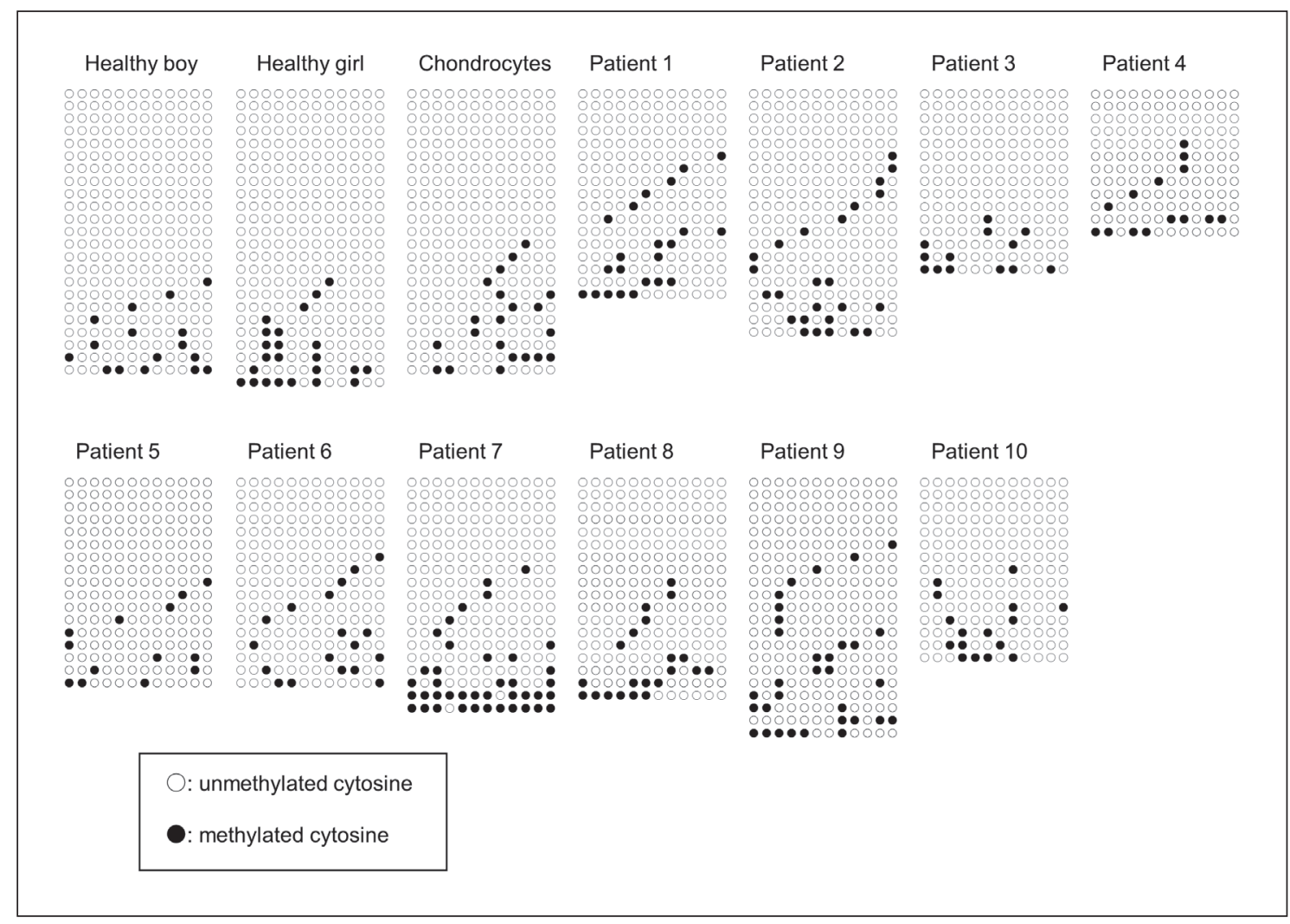

Fig. 4. Results of bisulfite sequencing. Twelve $\mathrm{CpG}$ dinucleotides in $\mathrm{CpG}$ island 1 were examined by Sanger sequencing. The results of genomic DNA samples obtained from blood cells of 2 healthy children and patients 1-10 and a DNA sample from chondrocytes of a healthy boy are shown.

In conclusion, the present study, in conjunction with our previous work, indicates that $\mathrm{CpG}$ islands in the SHOX-flanking region remain hypomethylated in healthy individuals as well as in ISS/LWD patients with pseudoautosomal CNVs, but can be methylated in women with extensive X-chromosomal rearrangements. Copy number changes of SHOX enhancers likely disturb SHOX expression through DNA methylation-independent mechanisms, which need to be determined in future studies.

\section{Statement of Ethics}

The study was approved by the Institutional Review Board Committee at the National Center for Child and Development and performed after obtaining informed consent from the participants or their parents.

\section{Disclosure Statement}

The authors declare no conflicts of interest.

\section{Funding Sources}

This study was supported by a research grant from JCR Pharmaceuticals to M.F. The sponsor had no role in the study design, in the collection, analysis or interpretation of data, in the writing of the report or in the decision to submit the report for publication.

References

Benito-Sanz S, del Blanco DG, Aza-Carmona M, Magano LF, Lapunzina P, et al: PAR1 deletions downstream of SHOX are the most frequent defect in a Spanish cohort of LériWeill dyschondrosteosis (LWD) probands. Hum Mutat 27:1062 (2006).

-Benito-Sanz S, Royo JL, Barroso E, PaumardHernández B, Barreda-Bonis AC, et al: Identification of the first recurrent PAR1 deletion in Léri-Weill dyschondrosteosis and idiopathic short stature reveals the presence of a novel SHOX enhancer. J Med Genet 49:442450 (2012).

- Berletch JB, Yang F, Xu J, Carrel L, Disteche CM: Genes that escape from $\mathrm{X}$ inactivation. Hum Genet 130:237-245 (2011). 
Bunyan DJ, Taylor EJ, Maloney VK, Blyth M: Homozygosity for a novel deletion downstream of the SHOX gene provides evidence for an additional long range regulatory region with a mild phenotypic effect. Am J Med Genet A 164A:2764-2768 (2014).

-Carrel L, Willard HF: X-inactivation profile reveals extensive variability in X-linked gene expression in females. Nature 434:400-404 (2005).

-Chen J, Wildhardt G, Zhong Z, Röth R, Weiss B, et al: Enhancer deletions of the SHOX gene as a frequent cause of short stature: the essential role of a $250 \mathrm{~kb}$ downstream regulatory domain. J Med Genet 46:834-839 (2009).

-Clement-Jones M, Schiller S, Rao E, Blaschke RJ, Zuniga A, et al: The short stature homeobox gene SHOX is involved in skeletal abnormalities in Turner syndrome. Hum Mol Genet 9: 695-702 (2000).

Deaton AM, Bird A: CpG islands and the regulation of transcription. Genes Dev 25:10101022 (2011).

-Durand C, Bangs F, Signolet J, Decker E, Tickle C, Rappold G: Enhancer elements upstream of the SHOX gene are active in the developing limb. Eur J Hum Genet 18:527-532 (2010).

-Flanagan SF, Munns CF, Hayes M, Williams B, Berry M, et al: Prevalence of mutations in the short stature homeobox containing gene (SHOX) in Madelung deformity of childhood. J Med Genet 39:758-763 (2002).
Fukami M, Kato F, Tajima T, Yokoya S, Ogata T: Transactivation function of an approximately 800 -bp evolutionarily conserved sequence at the SHOX $3^{\prime}$ region: implication for the downstream enhancer. Am J Hum Genet 78: 167-170 (2006).

Fukami M, Naiki Y, Muroya K, Hamajima T, Soneda S, et al: Rare pseudoautosomal copynumber variations involving SHOX and/or its flanking regions in individuals with and without short stature. J Hum Genet 60:553-556 (2015).

Funari MF, Jorge AA, Souza SC, Billerbeck AE, Arnhold IJ, et al: Usefulness of MLPA in the detection of SHOX deletions. Eur J Med Genet 53:234-238 (2010).

Nasu M, Takayama S, Umezawa A: Endochondral ossification model system: designed cell fate of human epiphyseal chondrocytes during long-term implantation. J Cell Physiol 230: 1376-1388 (2015).

Plath K, Mlynarczyk-Evans S, Nusinow DA, Panning B: Xist RNA and the mechanism of $\mathrm{X}$ chromosome inactivation. Annu Rev Genet 36:233-278 (2002).

- Poggi H, Vera A, Avalos C, Lagos M, Mellado C, et al: A deletion of more than $800 \mathrm{~kb}$ is the most recurrent mutation in Chilean patients with SHOX gene defect. Horm Res Paediatr 84:254-257 (2015).

Rao E, Weiss B, Fukami M, Rump A, Niesler B, et al: Pseudoautosomal deletions encompassing a novel homeobox gene cause growth failure in idiopathic short stature and Turner syndrome. Nat Genet 16:54-63 (1997).
Rosilio M, Huber-Lequesne C, Sapin H, Carel JC, Blum WF, Cormier-Daire V: Genotypes and phenotypes of children with SHOX deficiency in France. J Clin Endocrinol Metab 97:E1257E1265 (2012).

-Sandoval GT, Jaimes GC, Barrios MC, Cespedes C, Velasco HM: SHOX gene and conserved noncoding element deletions/duplications in Colombian patients with idiopathic short stature. Mol Genet Genomic Med 2:95-102 (2014).

Schneider KU, Sabherwal N, Jantz K, Röth R, Muncke N, et al: Identification of a major recombination hotspot in patients with short stature and SHOX deficiency. Am Hum Genet 77:89-96 (2005)

- Shima H, Tanaka T, Kamimaki T, Dateki S, Muroya $\mathrm{K}$, et al: Systematic molecular analyses of SHOX in Japanese patients with idiopathic short stature and Leri-Weill dyschondrosteosis. J Hum Genet 61:585-591 (2016).

Suzuki E, Shima H, Toki M, Hanew K, Matsubara $\mathrm{K}$, et al: Complex X-chromosomal rearrangements in two women with ovarian dysfunction: implications of chromothripsis/chromoanasynthesis-dependent and -independent origins of complex genomic alterations. Cytogenet Genome Res 150:86-92 (2016).

-Verdin H, Fernández-Miñán A, Benito-Sanz S, Janssens S, Callewaert B, et al: Profiling of conserved non-coding elements upstream of SHOX and functional characterisation of the SHOX cis-regulatory landscape. Sci Rep 5: 17667 (2015). 